\title{
Performance of interferon- $y$ release assay in the diagnosis of tuberculous lymphadenitis: a meta-analysis
}

\author{
Qianqian Liu ${ }^{1,2}$, Wenzhang Li ${ }^{3}$, Yufeng Chen ${ }^{1}$, Xinmiao Du ${ }^{2}$, Chengdi Wang ${ }^{2}$, Binmiao Liang ${ }^{2}$, Yin Tang \\ ${ }^{4,5}$, Yulin Feng ${ }^{2}$, Chuanmin Tao ${ }^{6}$, Jian-Qing He ${ }^{\text {Corresp. }} 2$ \\ 1 Department of Respiratory Diseases, Chengdu Municipal First People's Hospital, Chengdu, Sichuan, China \\ 2 Department of Respiratory and Critical Care Medicine, West China Hospital, Sichuan University, Chengdu, Sichuan, China \\ 3 Department of Cardiology, First Affiliated Hospital of Chengdu Medical College, Chengdu, Sichuan, China \\ 4 State Key Laboratory of Oral Disease, West China School \& Hospital of Stomotology, Sichuan University, Chengdu, Sichuan, China \\ 5 Herman Ostrow School of Dentistry, University of Southern California, Los Angeles, California, USA \\ 6 Department of Laboratory Medicine, West China Hospital, Sichuan University, Chengdu, Sichuan, China \\ Corresponding Author: Jian-Qing He \\ Email address: jianqhe@gmail.com
}

Background. The diagnostic values of interferon-gamma release assays (IGRA) in tuberculosis (TB) vary a lot with different site of infections, with especially higher sensitivities in chronic forms of TB, such as tuberculosis of lymph node. We conducted a meta-analysis to comprehensively evaluate the overall accuracy of diagnostic IGRA for tuberculous lymphadenitis.

Methods. Pubmed, Web of science, EMBASE, Wanfang and CNKI databases up to February 17, 2017 were searched to identify published studies. The study quality was evaluated using the QUADAS-2 checklist. The pooled estimates of diagnostic parameters were generated using a bivariate randomeffects model and summary receiver operating characteristic (SROC) curves were used to summarize global performance.

Results. A total of ten qualified studies, performed in Korea or China, including 1084 patients, were enrolled in this meta-analysis. The pooled estimates of diagnostic accuracy were as follows: sensitivity, 0.89 (95\% $0.85-0.92)$; specificity, 0.81 (95\% $0.77-0.83)$; positive likelihood ratio (PLR), $4.25(95 \% \mathrm{Cl}$ 2.79-6.47); negative likelihood ratio (NLR), $0.16(95 \% \mathrm{Cl} 0.12-0.22)$; and area under the curve (AUC) was 0.93. According to subgroup analyses, studies conducted using QuantiFERON-TB, in Korean population and focusing on cervical lymphadenitis exhibited relative higher specificity while lower sensitivity. No evidence of publication bias was identified.

Conclusions. IGRA exhibits high diagnostic accuracy in tuberculous lymphadenitis. The diagnostic value of IGRA differed by different IGRA methods, ethnicity and lymphadenitis location. Our conclusion may be more applicable to population from TB prevalent areas. 
1 Title

2 Performance of interferon- $\gamma$ release assay in the diagnosis of tuberculous lymphadenitis: a meta-

3 analysis

\section{Authors}

5 Qianqian Liu ${ }^{1,2 *}$, Wenzhang $\mathrm{Li}^{3 *}$, Yunfeng Chen ${ }^{1}$, Xinmiao $\mathrm{Du}^{2}$, Chengdi Wang ${ }^{2}$, Binmiao

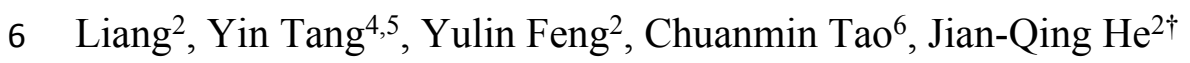

7 1. Department of Respiratory Diseases, Chengdu Municipal First People's Hospital, Chengdu, 8 Sichuan, China.

9 2. Department of Respiratory and Critical Care Medicine, West China Hospital, Sichuan 10 University, Chengdu, Sichuan, China.

11 3. Department of Cardiology, First Affiliated Hospital of Chengdu Medical College, Chengdu, 12 Sichuan, China.

13 4. State Key Laboratory of Oral Disease, West China School \& Hospital of Stomotology, 14 Sichuan University, Chengdu, Sichuan, China.

15 5. Herman Ostrow School of Dentistry, University of Southern California, Los Angeles, 16 California, USA.

17 6. Department of Laboratory Medicine, West China Hospital, Sichuan University, Chengdu, 18 Sichuan, China. 
$19 *$ These authors contributed equally.

$20 \uparrow$ Corresponding author:

21 Dr. Jian-Qing He

22 Address: Department of Respiratory and Critical Care Medicine, West China Hospital, Sichuan

23 University, 37\# Guo-xue-xiang, Chengdu 610041, Sichuan Province, China.

24 Tel: 01186-18980602293

25 Fax: 01186-85422571

26 E-mail: jianqhe@gmail.com 


\section{Abstract}

28 Background. The diagnostic values of interferon-gamma release assays (IGRA) in tuberculosis

29 (TB) vary a lot with different site of infections, with especially higher sensitivities in chronic 30 forms of $\mathrm{TB}$, such as tuberculosis of lymph node. We conducted a meta-analysis to 31 comprehensively evaluate the overall accuracy of diagnostic IGRA for tuberculous 32 lymphadenitis.

33 Methods. Pubmed, Web of science, EMBASE, Wanfang and CNKI databases up to February 17, 342017 were searched to identify published studies. The study quality was evaluated using the 35 QUADAS-2 checklist. The pooled estimates of diagnostic parameters were generated using a 36 bivariate random-effects model and summary receiver operating characteristic (SROC) curves 37 were used to summarize global performance.

Results. A total of ten qualified studies, performed in Korea or China, including 1084 patients, were enrolled in this meta-analysis. The pooled estimates of diagnostic accuracy were as follows: sensitivity, 0.89 (95\%CI 0.85-0.92); specificity, 0.81 (95\%CI 0.77-0.83); positive likelihood ratio (PLR), 4.25 (95\%CI 2.79-6.47); negative likelihood ratio (NLR), 0.16 (95\%CI 0.12-0.22); and area under the curve (AUC) was 0.93 . According to subgroup analyses, studies conducted using QuantiFERON-TB, in Korean population and focusing on cervical lymphadenitis exhibited relative higher specificity while lower sensitivity. No evidence of publication bias was identified. 
46 diagnostic value of IGRA differed by different IGRA methods, ethnicity and lymphadenitis

47 location. Our conclusion may be more applicable to population from TB prevalent areas. 
Introduction

49 Despite the advances in effective chemotherapy regimens and slight decrease of incidence, 50 tuberculosis (TB) still remains as a great challenge to public health. According to the most recent

51 World Health Organization (WHO) report, TB has surpassed acquired immune deficiency

52 syndrome (AIDS), becoming the leading cause of death from infectious disease, with an estimate

53 of 9.6 million new cases and 1.5 million deaths every year (WHO. Global tuberculosis report

54 2015. Geneva). Moreover, the decline of extrapulmonary tuberculosis (EPTB) is regarded as

55 suboptimal, and the proportion of EPTB among total TB cases has been increasing annually

56 (Barry et al. 2012; Peto et al. 2009; Sandgren et al. 2013). Among all EPTB, tuberculous

57 lymphadenitis, manifested as part of systemic processes or a unique lesion localized to lymph

58 node, is the most common form in many areas, accounting for around $20-50 \%$ of total EPTB

59 cases (Gonzalez et al. 2003; Ilgazli et al. 2004; Wiwatworapan \& Anantasetagoon 2008). The

60 characteristic local impaired presentation, such as spontaneous fistula to the skin, was noted in

61 only $4-11 \%$ of tuberculous lymphadenitis cases, and those classic TB systemaic signs, such as

62 prolonged fever, night sweats and anorexia et al. were also not frequent, compared with

63 pulmonary TB. Thus the clinical physical findings of tuberculous lymphadenitis are usually

64 indistinguishable from lymphadenitis caused by other etiologies, posing great diagnostic

65 challenges to clinician, and giving it great potential for delayed treatment (Fontanilla et al. 2011;

66 Handa et al. 2012; Yoon et al. 2004).

67 Up till now, several diagnostic choices are available for tuberculous lymphadenitis. Among 
68 them, the radiographic findings are frequently inconclusive and are merely clues for 69 diagnosis(Brodie \& Schluger 2005). Tuberculin skin test (TST) cannot distinguish 70 mycobacterium tuberculosis (MTB) infection from non-tuberculous mycobacteria (NTM) 71 infection, as well as Bacillus Calmette-Guerin (BCG) vaccine, thus limiting its diagnostic 72 application. Microbiologic and pathologic methods are usually the last procedures of choice, 73 however, they can only be performed through invasive operation. What's more, the 74 paucibacillary nature of the sample with non-uniform distributed bacilli often decreased the 75 sensitivity of microbiologic diagnostic tests (Brodie \& Schluger 2005). Also, the highly variable 76 performance of rapid PCR assay and non-specificity of histological examination usually leave 77 the diagnosis of tuberculous lymphadenitis unsettled (Hirunwiwatkul et al. 2002; Manitchotpisit 78 et al. 1999).

Recent years an immune-based blood assay, interferon- $\gamma$ (IFN- $\gamma$ ) release assay (IGRA), has been introduced for the diagnosis of TB based on the detection of IFN- $\gamma$ secreted by $\mathrm{T}$ cells 81 stimulated by two MTB-specific antigens, early secretory antigenic target (ESAT)-6 and culture 82 filtrate protein (CFP)-10. The advantage of IGRA over TST depends on its lack of cross83 reactivity with BCG vaccine strains and most NTM species (Berthet et al. 1998; Pai et al. 2014;

84 Pai et al. 2007). It is reported that the diagnostic value of the IGRA may differed by clinical 85 courses and site of infections, with especially higher sensitivities in chronic forms of TB, such as 86 lymph node TB (sensitivity $89 \%$ ) than in acute forms of TB (i.e., TB meningitis, sensitivity $74 \%$ ) 87 (Cho et al. 2011). The relative higher sensitivities among tuberculous lymphadenitis may be 
88

89

explained by more efficient immune response allowing for the detection of IFN- $\gamma$ production in those patients (Pai et al. 2006). Over the past few years, a growing number of studies investigating the diagnostic accuracy of IGRA for tuberculous lymphadenitis have come out with inconsistent results, with sensitivity ranging from 0.81 to 0.95 and specificity ranging from 0.52 to 0.96 (Kim et al. 2013; Kim et al. 2016; Liao et al. 2009). The contradiction of previous studies may be partly due to small sample size of individual studies which are lack of statistical power. To get robust evidence guiding clinicians on the diagnostic accuracy of IGRA in the diagnosis of tuberculous lymphadenitis, we conducted the present meta-analysis.

\section{Materials \& Methods}

\section{Publication search}

Five databases were searched from their inception until February 17, 2017: Pubmed, Web of science, EMBASE, Wanfang and CNKI databases. The search strategy included the following terms: 'elispot' OR 'quantiferon*' OR 'interferon-gamma release assay' OR 'interferon-gamma release assays' OR 'interferon-gamma releasing assay' OR 'interferon-gamma releasing assays' OR ‘t cell assay' OR 't cell assays' OR 'IGRA*' OR 'T.SPOT*' OR 'TSPOT*' OR 'T-SPOT*' in combination with 'lymphatic' OR 'lymph nodes' OR 'lymph node' OR 'lymphadenitis' in combination with 'diagnos*' OR 'sensitivity'. Furthermore, a manual search for relevant studies in reference list of identified articles was also conducted. No language restrictions were applied in our study. Studies were included in this meta-analysis if they met the following criteria: (1) IGRA was used to test for the diagnosis of tuberculous lymphadenitis; (2) diagnostic $2 \times 2$ tables 
108 (the value of true positive, false positive, false negative, and true negative) were reported or 109 could be calculated from original articles; (3) bacteriological findings or clinical response to anti110 tuberculosis therapy were used as reference standard test. The following studies were excluded:

111 (1) review or meta-analysis; (2) studies available only as abstracts; (3) duplicate publication of 112 previous research.

Data extraction

114 Two reviewers (Qianqian Liu and Wenzhang Li) independently screened those identified articles

115 by titles and abstracts. Then the full texts were evaluated for eligibility based on pre-set inclusion 116 and exclusion criteria. Discrepancies were resolved by discussion. The following variables were 117 extracted from all included studies: first author, language, publication year, country, sample size, 118 IGRA methods, IGRA cut-off values, and diagnostic 2x2 table values. Indeterminate results may 119 occur in both IGRA tests and excluding these patients may lead our results to increased 120 sensitivity and specificity. Therefore, those patients with indeterminate results were regarded as 121 negative results in all analyses.

\section{Quality assessment}

123 The revised Quality Assessment of Diagnostic Accuracy Studies (QUADAS-2) was used to 124 assess the study quality focusing on the risk of bias and the applicability (Whiting et al. 2011). 125 Any discrepancies by two reviewers were resolved by consensus. 
127 Heterogeneity was assessed by the Chi-squared test and $\mathrm{I}^{2}$ test, setting $\mathrm{P}<0.10$ as the threshold

128 for significance. The following accuracies with their 95\% confidence interval (CI) were pooled

129 respectively: sensitivity, specificity, positive likelihood ratio (PLR), negative likelihood ratio

130 (NLR), using bivariate random-effects model. Summary receiver operating characteristic (SROC)

131 curves were also generated. The Spearman rank correlation was conducted to test for potential

132 threshold effect, setting $\mathrm{P}<0.05$ as threshold for significance. Presence of publication bias was

133 assessed by Deeks' funnel plot with $\mathrm{P}<0.10$ for the slope coefficient suggested significant bias

134 (Deeks et al. 2005). All statistical tests were two-sided. Stata 12.0 (StataCorp, College Station,

135 TX, USA) and Meta-DiSc 1.4 were used for statistical analysis.

\section{Results}

\section{Study characteristics}

We initially identified 2875 relevant articles and a total of ten studies meeting inclusion criteria were included in the final meta-analysis (Fig. 1) (Cho et al. 2011; Jia et al. 2016; Jia et al. 2014; Kim et al. 2013; Kim et al. 2016; Kim et al. 2011; Liao et al. 2009; Lu et al. 2016; Shin et al. 2015; Song et al. 2009). The basic characteristics of each study are presented in Table 1 . The ten studies were conducted from 2009 to 2016, including 1084 patients distributed in China (4 studies) and Korea (6 studies) respectively. Two studies were published in Chinese language, one in Korean language and the remaining were in English. The adopted IGRA methods were QuantiFERON.TB Gold In Tube (QFT-GIT; Cellestis, Carnegie, VIC, Australia) in five studies and T-SPOT.TB (Oxford Immunotech, Abingdon, UK) in the other five studies. 
147 Quality assessments of the eligible studies

148 The quality assessments of the ten eligible studies are listed in Table 2. The risk bias of selection

149 domain was categorized as high in two studies, as they excluded patients with clinical diagnosed

150 tuberculous lymphadenitis, which may exaggerate diagnostic accuracy of index test. Only one

151 study had low risk bias of index test as it was interpreted without knowledge of the other test,

152 while the other studies did not report relevant information. As for the risk bias of references

153 standard test, two studies had low risk, one study had high risk and the others were scored

154 unknown. The risk bias of flow and timing domains and the concerns of applicability in all

155 domains were categorized as low.

156 Diagnostic performance

157 Chi-square values demonstrated no significant between-study heterogeneity among the following

158 diagnostic parameters: sensitivity, $5.38(\mathrm{P}=0.80)$ and NLR, $5.14(\mathrm{P}=0.82)$, while significant

159 heterogeneity was found for specificity $(\mathrm{P}<0.10)$ and PLR $(\mathrm{P}<0.10)$. The pooled estimates of 160 diagnostic accuracy were listed as follows (Fig. 2): sensitivity, 0.89 (95\%CI 0.85-0.92);

161 specificity, 0.81 (95\%CI 0.77-0.83); PLR, 4.25 (95\%CI 2.79-6.47); NLR, 0.16 (95\%CI 0.12-

162 0.22). Figure 3 shows the SROC curve and the area under the curve (AUC) was 0.93.

163 Threshold effect and subgroup analyses

164 If sensitivity and specificity were negatively correlated and ROC exhibited typical 'shoulder 165 arm-shaped' distribution, then threshold effect should be considered. Our statistical results 
166 showed no significant threshold effect, with the spearman correlation coefficient to be 0.10 $167(\mathrm{P}=0.78)$.

Subgroup analyses by IGRA methods, ethnicity and tuberculous lymphadenitis locations were performed to identify potential sources of heterogeneity (Table 3). In general, the values of AUC in all subgroup analyses were more than 0.9 without exception. When stratified by IGRA methods, T-SPOT.TB had a relatively higher sensitivity and lower specificity than QuantiFERON-TB. While when stratified by ethnicity, studies conducted in Chinese population had a relatively higher sensitivity and lower specificity than those in Korean population. As some studies included patients with lymphadenitis localizing to cervical areas while other studies didn't restrict the location of lymph nodes, which may also lead to variability in diagnostic accuracy. Our subsequent subgroup analysis showed that limiting lymph nodes to cervical areas exhibited lower sensitivity and higher specificity compared with other studies.

Publication bias

Deeks' funnel plots was symmetric and the bias coefficient was not significant $(P=0.54)$ (Fig. 4).

Our evaluation found no evidence of publication bias.

\section{Discussion}

The IGRA, an in vitro immunodiagnostic test, is being adopted increasingly for the detection of

MTB infection and has generated promising results as an alternative diagnostic tool for the LTBI

184 (Diel et al. 2011). However, the application of this newly emerging technique may not only be 
185

186

187

188

189

190

191

192

193

194

195

196

197

198

199

200

201

202

203

204

exclusive to LTBI. Several recent studies have investigated the diagnostic value of IGRA upon EPTB according to different sites of infection and uniformly reported a higher sensitivity of IGRA in tuberculous lymphadenitis detection than in other form of EPTB (Cho et al. 2011; Song et al. 2009). Thus we conducted the present meta-analysis to comprehensively evaluate the overall diagnostic accuracy of IGRA upon tuberculous lymphadenitis.

$$
\text { The pooling results showed that the sensitivity and specificity of IGRA was } 0.89 \text { and } 0.81
$$

respectively, which means that $11 \%$ patients would have a missed diagnosis of tuberculous lymphadenitis and 19\% would be falsely diagnosed as tuberculous lymphadenitis if we use IGRA as the only diagnostic method. The pooled PLR and NLR of IGRA was 4.25 and 0.16 respectively, which indicates that patients with tuberculous lymphadenitis have 4.25 times of chance getting a positive result than those without tuberculous lymphadenitis and those with tuberculous lymphadenitis have 0.16 times chance of getting negative result than those without tuberculous lymphadenitis. AUC is a comprehensive index combining the sensitivity and specificity. Generally, AUC $>0.9$ is regarded to be highly accurate in diagnostic tests (Deeks 2001). Accordingly, our summarized result of AUC (0.93) indicated high diagnostic accuracy. As meta-analysis is a pooling result of previous individual studies with larger statistical power, our conclusion is more reliable than previous small sample sized individual studies.

The subgroup results showed T-SPOT.TB had a relatively higher sensitivity and lower specificity than QuantiFERON-TB, which is in accordance with previous reports. Lee et al. conducted a head-to-head comparison between the two assays in the diagnosis of MTB infection. 
205 They reported higher sensitivity and inferior specificity of T-SPOT.TB over QuantiFERON-TB

206 (Lee et al. 2006). The same tendency in diagnosing active tuberculosis was found by another

207 study (Lai et al. 2011). It may be attributed to the fact that the QFT-GIT assay tends to be more

208 easily affected by immune status and peripheral lymphocyte counts or simply be related to the

209 pre-set cut-off values of the two commercial assays (Komiya et al. 2010; Lee et al. 2006).

210 Although we detected the same tendency with previous studies, our research focused on

tuberculous lymphadenitis, which is different from previous studies not focusing on this special

212 type of tuberculosis. When stratified by ethnicity, those studies conducted in Chinese population

213 had a relatively higher sensitivity and lower specificity than which in Korean population. Such

214 similar tendency may be partially explained by ethnic difference or by the fact that all studies

215 conducted in Chinese population of this meta-analysis were performed using T-SPOT.TB while

216 all but one study conducted in Korean population were performed using QuantiFERON-TB. Our

217 subgroup analysis also showed that studies with lymphadenitis localizing to cervical areas

218 exhibited lower sensitivity and higher specificity compared with studies which didn't restrict the

219 location of lymph nodes. Whether there is a biological basis or it is just a statistical coincidence

220 need to be further determined.

As for QUADAS-2 assessment, none of our included studies had low risk of bias in all

222 domains. In patient selection domain, eight studies scored unclear because they didn't clearly

223 state whether they enrolled sample of patients consecutively or randomly. What's more, in index

224 test and reference standard test domains, most studies did not report whether the index test or 
225 reference standard test were interpreted without knowledge of the other test. It is important to

226 note that low quality original research may introduce heterogeneity in meta-analysis. Thus, it

227 needs to be stressed that the future diagnostic studies should be reported in detail according to

228 relevant diagnostic study guidelines.

After all, some limitations should be pointed out. First, as most of patients included in the

230 present study were immunocompetent, caution is advised when applying our conclusion to

231 immunocompromised patients. Second, despite our thorough literature searching without

232 language limitation, all studies of this meta-analysis were conducted either in Korea or in China,

233 both were TB high prevalence areas. As we know, the diagnostic parameters, such as sensitivity

234 and specificity, may be affected by the prevalence of the target disease. Thus, our conclusion

235 might be more appropriate when used in TB high-burden areas.

236 Conclusion

237 To sum up, IGRA exhibits high diagnostic accuracy in tuberculous lymphadenitis and may play

238 an increasing important role as a supplementary diagnostic tool with the advantage of

239 noninvasiveness and efficiency. The diagnostic value of IGRA differed by different IGRA

240 methods, ethnicity and lymphadenitis location. Our conclusion may be more applicable to

241 population from TB prevalent areas. 
242

243

244

245

246

247

248

249

250

251

252

253

254

255

256

257

258

259

260

261

262

263

264

265

266

267

268

269

270

\section{References}

Barry C, Waring J, Stapledon R, Konstantinos A, and National Tuberculosis Advisory Committee ftCDNA. 2012. Tuberculosis notifications in Australia, 2008 and 2009. Commun Dis Intell Q Rep 36:82-94.

Berthet FX, Rasmussen PB, Rosenkrands I, Andersen P, and Gicquel B. 1998. A Mycobacterium tuberculosis operon encoding ESAT-6 and a novel low-molecular-mass culture filtrate protein (CFP-10). Microbiology 144 ( Pt 11):3195-3203. 10.1099/00221287-144-11-3195

Brodie D, and Schluger NW. 2005. The diagnosis of tuberculosis. Clin Chest Med 26:247-271, vi. 10.1016/j.ccm.2005.02.012

Cho OH, Park KH, Kim SM, Park SJ, Moon SM, Chong YP, Sung H, Kim MN, Jeong JY, Lee SO, Choi SH, Woo JH, Kim YS, and Kim SH. 2011. Diagnostic performance of TSPOT.TB for extrapulmonary tuberculosis according to the site of infection. $J$ Infect 63:362-369. 10.1016/j.jinf.2011.06.010

Deeks JJ. 2001. Systematic reviews in health care: Systematic reviews of evaluations of diagnostic and screening tests. BMJ 323:157-162.

Deeks JJ, Macaskill P, and Irwig L. 2005. The performance of tests of publication bias and other sample size effects in systematic reviews of diagnostic test accuracy was assessed. $J$ Clin Epidemiol 58:882-893. 10.1016/j.jclinepi.2005.01.016

Diel R, Goletti D, Ferrara G, Bothamley G, Cirillo D, Kampmann B, Lange C, Losi M, Markova R, Migliori GB, Nienhaus A, Ruhwald M, Wagner D, Zellweger JP, Huitric E, Sandgren A, and Manissero D. 2011. Interferon-gamma release assays for the diagnosis of latent Mycobacterium tuberculosis infection: a systematic review and meta-analysis. Eur Respir $J 37: 88-99$. 10.1183/09031936.00115110

Fontanilla JM, Barnes A, von Reyn CF. 2011. Current diagnosis and management of peripheral tuberculous lymphadenitis. Clin Infect Dis 53:555-562.

Gonzalez OY, Adams G, Teeter LD, Bui TT, Musser JM, and Graviss EA. 2003. Extrapulmonary manifestations in a large metropolitan area with a low incidence of tuberculosis. Int J Tuberc Lung Dis 7:1178-1185.

Handa U, Mundi I, Mohan S. 2012. Nodal tuberculosis revisited: a review. J Infect Dev Ctries 
6:6-12.

Hirunwiwatkul P, Tumwasorn S, Chantranuwat C, and Sirichai U. 2002. A comparative study of diagnostic tests for tuberculous lymphadenitis: polymerase chain reaction vs histopathology and clinical diagnosis. J Med Assoc Thai 85:320-326.

Ilgazli A, Boyaci H, Basyigit I, and Yildiz F. 2004. Extrapulmonary tuberculosis: clinical and epidemiologic spectrum of 636 cases. Arch Med Res 35:435-441. 10.1016/j.arcmed.2004.05.008.

Jia H, Pan L, Du B, Sun Q, Wei R, Xing A, Du F, Sun H, and Zhang Z. 2016. Diagnostic performance of interferon-gamma release assay for lymph node tuberculosis. Diagn Microbiol Infect Dis 85:56-60. 10.1016/j.diagmicrobio.2016.02.001

Jia HY, Pan LP, Liu F, Du BP, Sun Q, Xing AY, Du FJ, Ma Y, and Zhang ZD. 2014. Adjunctive Diagnostic Value of Interferon-gamma Release Assay in Tuberculosis Lymphadenitis. Tuber \& Thor Tumor:167-171.

Kim JK, Ko JJ, and Kim KC. 2013. Comparison of the Tuberculin Skin Test and the Interferon- $\gamma$ Release Assay for the Diagnosis of Cervical Tuberculous Lymphadenitis. Korean $J$ Otorhinolaryngol-Head Neck Surg 56:354-358.

Kim KH, Kim RB, and Woo SH. 2016. The efficacy of the interferon-gamma release assay for diagnosing cervical tuberculous lymphadenitis: A prospective controlled study. Laryngoscope 126:378-384. 10.1002/lary.25540

Kim YK, Uh Y, Lee NS, Cho MY, Eom M, and Kim HY. 2011. Whole-blood interferon-gamma release assay for diagnosis of tuberculous lymphadenitis. Tohoku J Exp Med 224:189-193.

Komiya K, Ariga H, Nagai H, Teramoto S, Kurashima A, Shoji S, and Nakajima Y. 2010. Impact of peripheral lymphocyte count on the sensitivity of 2 IFN-gamma release assays, QFT-G and ELISPOT, in patients with pulmonary tuberculosis. Intern Med 49:18491855.

Lai CC, Tan CK, Lin SH, Liao CH, Huang YT, and Hsueh PR. 2011. Diagnostic performance of whole-blood interferon-gamma assay and enzyme-linked immunospot assay for active tuberculosis'. Diagn Microbiol Infect Dis 71:139-143. 10.1016/j.diagmicrobio.2011.05.013 
301

302

303

304

305

306

307

308

309

310

311

312

313

314

315

316

317

318

319

320

321

322

323

324

325

326

327

328

329

330

331

Lee JY, Choi HJ, Park IN, Hong SB, Oh YM, Lim CM, Lee SD, Koh Y, Kim WS, Kim DS, Kim WD, and Shim TS. 2006. Comparison of two commercial interferon-gamma assays for diagnosing Mycobacterium tuberculosis infection. Eur Respir $J$ 28:24-30. 10.1183/09031936.06.00016906

Liao CH, Chou CH, Lai CC, Huang YT, Tan CK, Hsu HL, and Hsueh PR. 2009. Diagnostic performance of an enzyme-linked immunospot assay for interferon-gamma in extrapulmonary tuberculosis varies between different sites of disease. J Infect 59:402-408. 10.1016/j.jinf.2009.10.001

Lu X, Li WP, Xie YH, Sun RL, Liu W, and Jin FG. 2016. Evaluation of interferon-gamma release technique in extra-pulmonary tuberculosis detection. Chin J Lung Dis(Electronic Edition) 9:20-25.

Manitchotpisit B, Kunachak S, Kulapraditharom B, and Sura T. 1999. Combined use of fine needle aspiration cytology and polymerase chain reaction in the diagnosis of cervical tuberculous lymphadenitis. J Med Assoc Thai 82:363-368.

Pai M, Denkinger CM, Kik SV, Rangaka MX, Zwerling A, Oxlade O, Metcalfe JZ, Cattamanchi A, Dowdy DW, Dheda K, and Banaei N. 2014. Gamma interferon release assays for detection of Mycobacterium tuberculosis infection. Clin Microbiol Rev 27:3-20. 10.1128/CMR.00034-13

Pai M, Dheda K, Cunningham J, Scano F, and O'Brien R. 2007. T-cell assays for the diagnosis of latent tuberculosis infection: moving the research agenda forward. Lancet Infect Dis 7:428-438. 10.1016/S1473-3099(07)70086-5

Pai M, Kalantri S, and Menzies D. 2006. Discordance between tuberculin skin test and interferon-gamma assays. Int J Tuberc Lung Dis 10:942-943.

Peto HM, Pratt RH, Harrington TA, LoBue PA, and Armstrong LR. 2009. Epidemiology of extrapulmonary tuberculosis in the United States, 1993-2006. Clin Infect Dis 49:13501357. $10.1086 / 605559$

Sandgren A, Hollo V, and van der Werf MJ. 2013. Extrapulmonary tuberculosis in the European Union and European Economic Area, 2002 to 2011. Euro Surveill 18.

Shin JA, Chang YS, Kim HJ, Ahn CM, and Byun MK. 2015. Diagnostic utility of interferongamma release assay in extrapulmonary tuberculosis. Diagn Microbiol Infect Dis 82:4448. 10.1016/j.diagmicrobio.2015.02.002 
332 Song KH, Jeon JH, Park WB, Kim SH, Park KU, Kim NJ, Oh MD, Kim HB, and Choe KW.

333

334

335

336

337

338

339

340

341

342

343

344

345

346 2009. Usefulness of the whole-blood interferon-gamma release assay for diagnosis of extrapulmonary tuberculosis. Diagn Microbiol Infect Dis 63:182-187. 10.1016/j.diagmicrobio.2008.10.013

Whiting PF, Rutjes AW, Westwood ME, Mallett S, Deeks JJ, Reitsma JB, Leeflang MM, Sterne JA, Bossuyt PM, and Group Q-. 2011. QUADAS-2: a revised tool for the quality assessment of diagnostic accuracy studies. Ann Intern Med 155:529-536. 10.7326/00034819-155-8-201110180-00009

WHO. Global tuberculosis report 2015. Geneva SW, 2015. http://www.who.int/tb/publications/global_report/en/(accessed July 1, 2016).

Wiwatworapan T, and Anantasetagoon T. 2008. Extra-pulmonary tuberculosis at a regional hospital in Thailand. Southeast Asian J Trop Med Public Health 39:521-525.

Yoon HJ, Song YG, Park WI, Choi JP, Chang KH, and Kim JM. 2004. Clinical manifestations and diagnosis of extrapulmonary tuberculosis. Yonsei Med $J$ 45:453-461. 10.3349/ymj.2004.45.3.453 
Figure 1

Flow diagram of included studies.

Records identified through database

searching

$(n=2875)$




Figure 2

Forest plot evaluating the sensitivity and specificity of diagnostic performance of IGRA in tuberculous lymphadenitis.

$\mathrm{Cl}$, confidence interval. (A) Sensivitity; (B) Specificity.

A

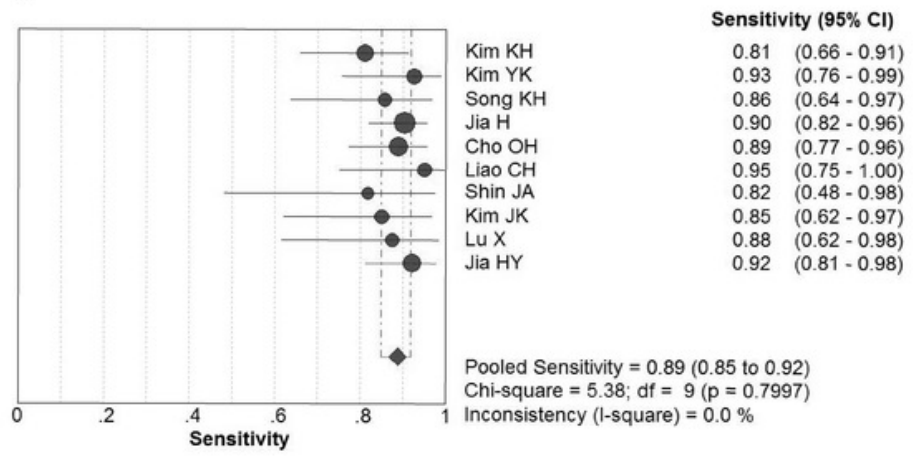

B

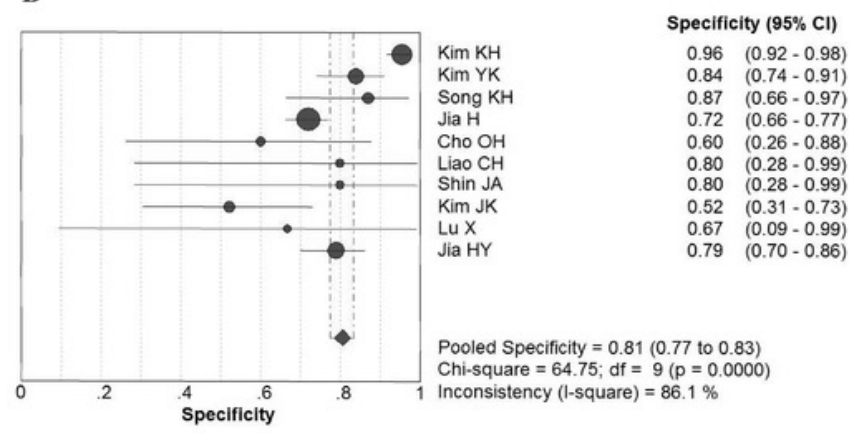


Figure 3

Summary receiver operator characteristics (SROC) of IGRA on summary estimates of sensitivity and specificity.

AUC, area under the curve; SE, standard error

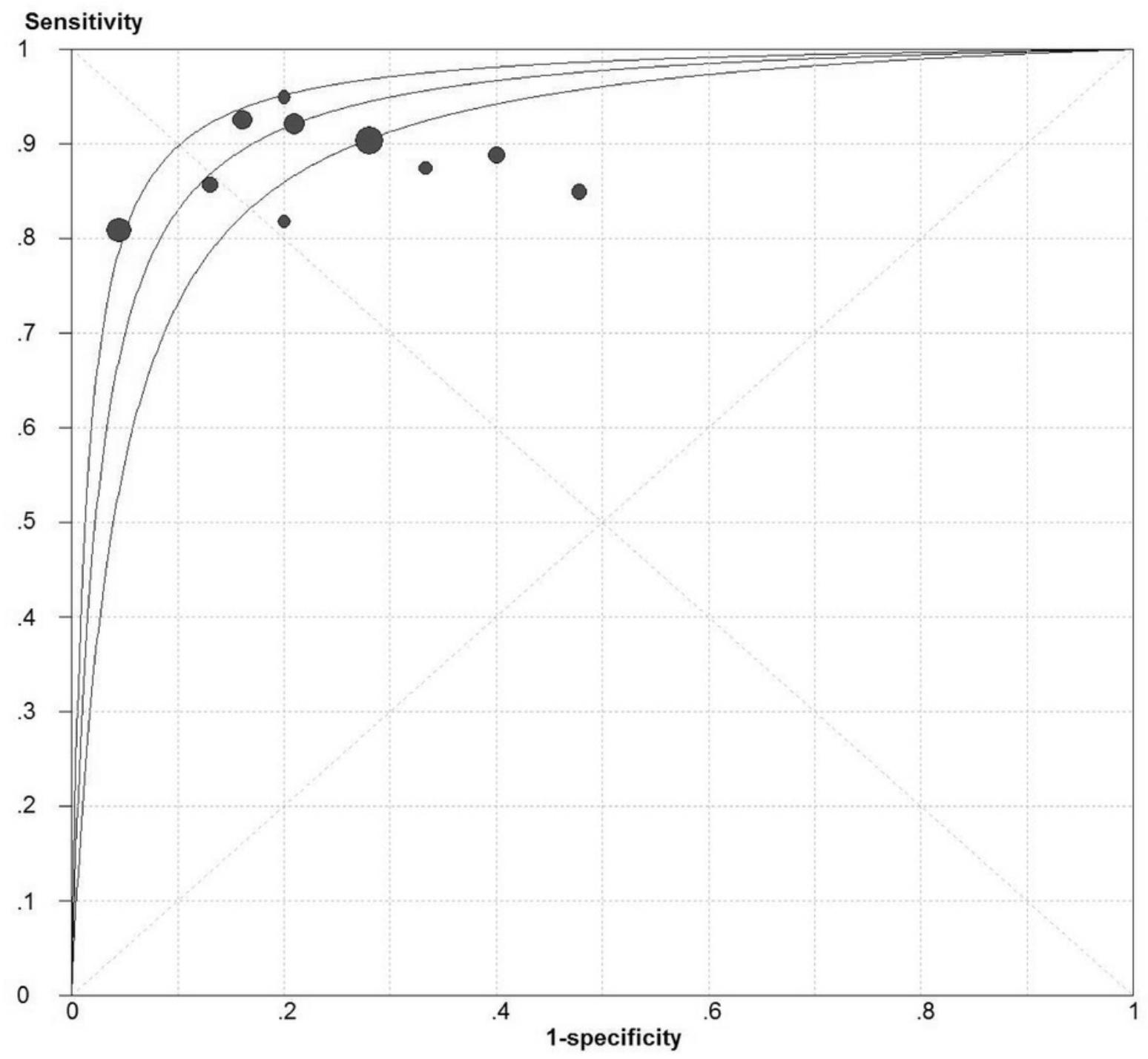

Symmetric SROC $A \cup C=0.9332$ $\mathrm{SE}(\mathrm{AUC})=0.0118$ $Q^{\star}=0.8689$ $\operatorname{SE}\left(Q^{*}\right)=0.0144$ 
Figure 4

Deeks' funnel plots for publication bias.

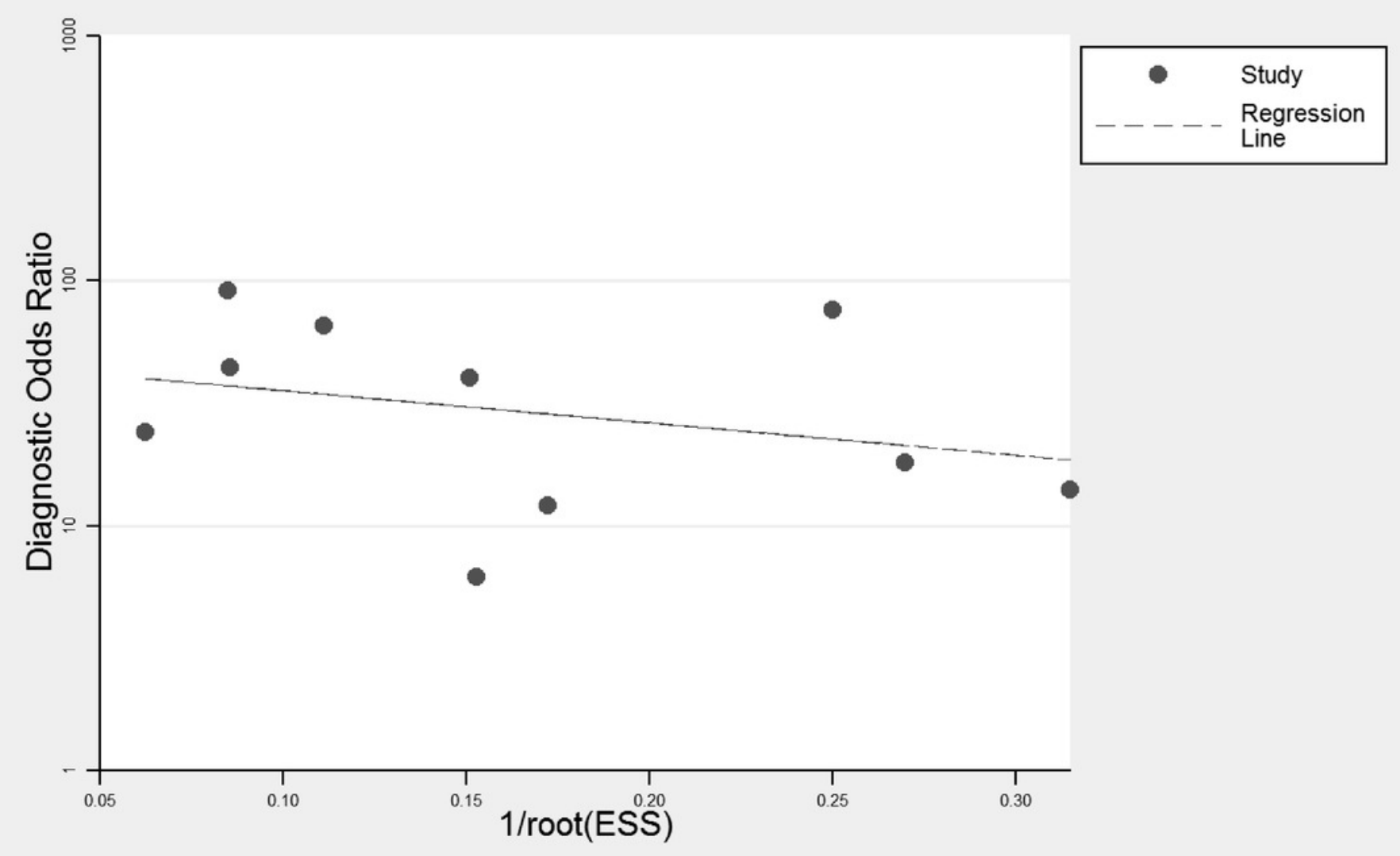




\section{Table $\mathbf{1}$ (on next page)}

Characteristics of studies included in meta-analysis.

IGRA, interferon- $\gamma$ release assay; FN, false negative; FP, false positive; TN, true negative; TP, true positive. 


\begin{tabular}{llllllllll}
\hline First author & Language & Year & Country & IGRA method & Sample size & TP & FP & FN & TN \\
\hline Kim KH & English & 2016 & Korea & QuantiFERON-TB & 244 & 34 & 9 & 8 & 193 \\
Kim YK & English & 2011 & Korea & QuantiFERON-TB & 108 & 25 & 13 & 2 & 68 \\
Song KH & English & 2009 & Korea & QuantiFERON-TB & 44 & 18 & 3 & 3 & 20 \\
Jia H & English & 2016 & China & T-SPOT.TB & 365 & 75 & 79 & 8 & 203 \\
Cho OH & English & 2011 & Korea & T-SPOT.TB & 64 & 48 & 4 & 6 & 6 \\
Liao CH & English & 2009 & China & T-SPOT.TB & 25 & 19 & 1 & 1 & 4 \\
Shin JA & English & 2015 & Korea & QuantiFERON-TB & 16 & 9 & 1 & 2 & 4 \\
Kim JK & Korean & 2013 & Korea & QuantiFERON-TB & 43 & 17 & 11 & 3 & 12 \\
Lu X & Chinese & 2016 & China & T-SPOT.TB & 19 & 14 & 1 & 2 & 2 \\
Jia HY & Chinese & 2014 & China & T-SPOT.TB & 156 & 47 & 22 & 4 & 83 \\
\hline
\end{tabular}




\section{Table 2 (on next page)}

Quality assessment of the eligible studies by QUADAS-2 


\begin{tabular}{|c|c|c|c|c|c|c|c|}
\hline \multirow[t]{2}{*}{ Study } & \multicolumn{4}{|c|}{ Risk of bias } & \multicolumn{3}{|c|}{ Applicability concerns } \\
\hline & Patient selection & Index test & Reference standard & Flow and timing & Patient selection & Index test & Reference standard \\
\hline 1 & unkown & unknown & unknown & low & low & low & low \\
\hline 2 & unkown & unknown & unknown & low & low & low & low \\
\hline 3 & unkown & unknown & high & low & low & low & low \\
\hline 4 & high & low & low & low & low & low & low \\
\hline 5 & unkown & unknown & low & low & low & low & low \\
\hline 6 & unknown & unknown & unknown & low & low & low & low \\
\hline 7 & unknown & unknown & unknown & low & low & low & low \\
\hline 8 & unknown & unknown & unknown & low & low & low & low \\
\hline 9 & unknown & unknown & unknown & low & low & low & low \\
\hline 10 & high & unknown & unknown & low & low & low & low \\
\hline
\end{tabular}




\section{Table 3(on next page)}

Subgroup analyses

PLR, positive likelihood ratio; NLR, negative likelihood ratio; AUC, area under the curve;TBL, Tuberculous lymphadenitis. 


\begin{tabular}{|c|c|c|c|c|c|c|c|c|c|c|}
\hline \multirow[t]{2}{*}{ Subgroup } & \multirow{2}{*}{$\begin{array}{l}\text { Studies } \\
\text { (n) }\end{array}$} & \multicolumn{2}{|c|}{ Sensitivity } & \multicolumn{2}{|c|}{ Specificity } & \multicolumn{2}{|l|}{ PLR } & \multicolumn{2}{|l|}{ NLR } & \multirow{2}{*}{$\begin{array}{c}\text { AUC } \\
\text { Pooled value }\end{array}$} \\
\hline & & Pooled value & $\mathrm{P}_{\text {Heter }}$ & Pooled value & $\mathrm{P}_{\text {Heter }}$ & Pooled value & $\mathrm{P}_{\text {Heter }}$ & Pooled value & $\mathrm{P}_{\text {Heter }}$ & \\
\hline T-SPOT.TB & 5 & $0.91(0.86-0.94)$ & 0.90 & $0.74(0.69-0.78)$ & 0.54 & $3.37(2.85-4.00)$ & 0.48 & $0.13(0.09-0.21)$ & 0.78 & $0.98(0.03)$ \\
\hline QuantiFERON-TB & 5 & $0.85(0.78-0.91)$ & 0.73 & $0.89(0.85-0.92)$ & $<0.10$ & $5.53(2.11-14.52)$ & $<0.10$ & $0.19(0.12-0.29)$ & 0.72 & $0.92(0.02)$ \\
\hline Korean & 6 & $0.86(0.80-0.91)$ & 0.77 & $0.88(0.84-0.91)$ & $<0.10$ & $4.71(2.08-10.67)$ & $<0.10$ & $0.19(0.13-0.28)$ & 0.84 & $0.92(0.02)$ \\
\hline Chinese & 4 & $0.91(0.86-0.95)$ & 0.85 & $0.74(0.69-0.78)$ & 0.53 & $3.45(2.90-4.11)$ & 0.50 & $0.12(0.07-0.20)$ & 0.80 & $0.96(0.13)$ \\
\hline Cervical TBL & 3 & $0.83(0.73-0.90)$ & 0.86 & $0.91(0.86-0.94)$ & $<0.10$ & $5.89(1.08-31.93)$ & $<0.10$ & $0.21(0.13-0.33)$ & 0.77 & $0.91(0.03)$ \\
\hline Not limit to Cervical TBL & 7 & $0.90(0.86-0.94)$ & 0.92 & $0.75(0.71-0.79)$ & 0.29 & $3.72(2.97-4.66)$ & 0.29 & $0.14(0.09-0.20)$ & 0.82 & $0.95(0.04)$ \\
\hline
\end{tabular}

1 